\title{
Microparticles and microplastics released from daily use of plastic feeding and water bottles and plastic injectors: potential risks to infants and children in China
}

\author{
Ke Song ${ }^{1,2} \cdot$ Runrun Ding ${ }^{1,3} \cdot$ Caiyun Sun ${ }^{1,3} \cdot$ Lunguang Yao ${ }^{1,3} \cdot$ Weicheng Zhang ${ }^{1,3}$ \\ Received: 26 March 2021 / Accepted: 12 June 2021 / Published online: 19 June 2021 \\ (C) The Author(s), under exclusive licence to Springer-Verlag GmbH Germany, part of Springer Nature 2021
}

\begin{abstract}
Daily use of plastic feeding and water bottles occur widely in China, and they could be sources for release of microplastics (MPs), which threaten the health of Chinese infants and children during daily usage. In this work, we investigated the use of polycarbonate (PC) and polypropylene (PP) for making water bottles (WBs) and polyphenylene sulfone resins (PPSU) for making feeding bottles (FBs), and we found that feeding bottles and water bottles released microparticles in amounts ranging from 53 to 393 particles/mL during 100 opening/closing cycles. The good linear regressions for plots of microparticles released vs. abrasion distance $\left(r^{2}=0.811\right)$ indicated that thick-necked bottles release more microparticles than thin-necked bottles. The brands and types of bottles (plastic vs. glass) influence microparticle release, and this indicates that high-quality plastic and glass bottles release fewer microparticles and are good for the health of infants and children. In addition to calcium stearate and silicone additives, the identified MPs account for 7.5 to $42.1 \%$ of released microparticles with different polymer types, sizes (from 20 to $500 \mu \mathrm{m}$ ) and shapes (cubic, spherical and irregular shapes). Additionally, an average of $1.74 \mathrm{MPs}$ were released from an injection with a single-use plastic injector. Nevertheless, a number of microparticles and nanosized plastics were observed with all samples, suggesting that the health risks of micro- and nanosized particles to humans, especially babies and children, and the environment should be considered seriously.
\end{abstract}

Keywords Microplastics $\cdot$ Human exposure $\cdot$ Plastic feeding bottles $\cdot$ Plastic water bottles $\cdot$ Plastic additives

\section{Introduction}

Microplastics (MPs) are an important aspect of global plastic pollution and are frequently detected in aquatic, soil and atmospheric environments (Gatidou et al. 2019; Prata et al. 2019; Rillig and Bonkowski 2018; Xiong et al. 2018), which

Responsible Editor: Lotfi Aleya

Weicheng Zhang

weichengzhang@nynu.edu.cn

1 Collaborative Innovation Center of Water Security for Water Source Region of the Midline of the South-to-North Diversion Project of Henan Province, Nanyang Normal University, Nanyang 473061, China

2 Ocean University of China, Qingdao 266100, China

3 Henan Key Laboratory of Ecological Security for Water Source Region of Midline of South-to-North Diversion Project, Nanyang Normal University, Nanyang 473061, China could pose a serious threat to environmental safety and human health (Alimba and Faggio 2019; Danopoulos et al. 2020; Guzzetti et al. 2018; Pagano et al. 2019; Prokić et al. 2021; Strungaru et al. 2019; Zhang et al. 2020). These existing MPs can be enriched by lettuce or ingested by zooplankton, fish, seabirds and humans (Botterell et al. 2019; Horton et al. 2018; Nelms et al. 2018; Watts et al. 2015). The MPs could result in a series of hazardous effects by promoting oxidative damage (lipid peroxidation and DNA strand damage), modifying antioxidative and metabolic systems (inhibiting superoxide dismutase, catalase and glutathione peroxidase) and inducing neurotoxicity (inhibiting acetylcholinesterase activity) (Burgos-Aceves et al. 2021; Du et al. 2020; Prokić et al. 2019; Sun et al. 2020). Due to the ecotoxicity of MPs for biota, the health implications of human exposure to MPs are drawing public attention. Indeed, MPs are known to be internalized in the gastrointestinal tract, and the unabsorbed portion is excreted with human faeces (Schwabl et al. 2019; Senathirajah et al. 2021). Therefore, the identification of the 
pathways by which MPs enter the human body forms a basis for evaluating their possibly hazardous effects.

The sources for MPs intake by humans are various and include table salt, drinking water, inhalation, food consumption and plastic products (such as plastic food containers, tea bags, feeding bottles and baby tools) (Ding et al. 2021; Fadare et al. 2020; Hernandez et al. 2019; Li et al. 2020; Peixoto et al. 2019; Senathirajah et al. 2021; Shruti et al. 2021; Uhl et al. 2018; Winkler et al. 2019). During the COVID-19 pandemic, masks are also a source of microplastic release (Aragaw 2020). These MPs include primary and secondary MPs with various concentrations, sizes and shapes, which are highly dependent on intake sources. It is likely that 3 to $38 \mathrm{mg}$ of primary MPs with cubic, spherical, rod-like and irregular shapes are consumed by humans or released into the environment during each use of a plastic food container in China (Fadare et al. 2020). Recently, polypropylene (PP) MPs were found to be released from feeding bottles in numbers as high as 16200 particles per millilitre after heat treatment (Li et al. 2020), suggesting that MPs may pose an unknown risk to infant health. These studies suggested that humans are subject to a serious and hazardous threat from MPs exposure. In addition to sources, various usage methods release significantly different amounts of MPs. Notably, sterilization and exposure to high-temperature water significantly increased PP MPs release from feeding bottles ( $\mathrm{Li}$ et al. 2020). Similarly, tea bags were identified as a source of MPs release (11.6 billion MPs and 3.1 billion nanoplastic particles (NPs) for a single cup of tea) (Hernandez et al. 2019) during 5 min of exposure to hot water $\left(95^{\circ} \mathrm{C}\right)$. The abrasion of plastic bottle caps against glass bottle bodies was identified as a source of large amounts of MPs (6300 items per litre) released from consumption of glass-bottled water (Uhl et al. 2018). However, different outcomes are proposed for these studies based on the different detection methods. For example, teabags are not believed by some authors to release billions of plastic particles (Busse et al. 2020; Hernandez et al. 2020), and mechanical stress (bottle openings/closings) is not considered a major source of MPs release during use of plastic water bottles (Winkler et al. 2019). Nevertheless, the release of MPs from the use of teabags and water bottles has been proven definitively (Busse et al. 2020; Hernandez et al. 2019), suggesting the risk of MPs exposure in humans.

Notably, the use of plastic feeding bottles and water bottles (FBs and WBs) is very popular with feeding infants and school students in China due to their durability, low cost and ready availability. To understand the possible risk of MPs exposure to Chinese children, we evaluated how feeding and water bottles act as MPs release sources during the bottle opening/closing process. Feeding bottles and water bottles with different brands and glass or plastic bodies capped with plastic caps were employed in this research. In addition, plastic injectors were also evaluated in this study due to their extensive use by diabetic patients and in antibiotic injections in livestock farms. A laser direct infrared (LDIR) chemical imaging system was used to identify polymer type and sizes for the collected particles.

\section{Materials and methods}

\section{Feeding and water bottles}

Feeding and water bottles were purchased from Taobao, a famous Chinese online shopping retail platform (https:// www.taobao.com/). The feeding and water bottles purchased involved different brands, prices, neck diameters and polymer types because different polymer types release different particles that may be hazardous to health. Sterile plastic injectors $(5 \mathrm{~mL})$ were also evaluated in this research. Detailed sample information is outlined in Table S1, and representative pictures of injectors are also given in SI (Figure S1). Ethanol was used to collect particle samples and was purchased from Tianjin Kemiou Chemical Reagent Co. Ltd., China ( $\geq 98 \%$ purity).

\section{Contamination prevention measures}

Ethanol was passed through a $0.22 \mu \mathrm{m}$ glass-fibre filter to exclude the possibility of plastic pollution. During sampling, plastic products were not used but were replaced by glass or aluminium containers. The feeding bottles or water bottles and their plastic caps, as well as the glass or aluminium containers, were washed twice with filtered ethanol and air dried on a vertical flow clean bench. The sampling processes were performed in a vertical flow clean bench to avoid plastic fibre contamination from the air.

\section{Sample collection}

One hundred opening/closing cycles were performed for each (glass or plastic) feeding bottle and water bottle, after which the ethanol was passed through a $0.22 \mu \mathrm{m}$ glass-fibre filter, and the bottleneck and caps were carefully washed three times with filtered ethanol $(10 \mathrm{~mL})$. One hundred plastic sterile injectors $(5 \mathrm{~mL})$ were washed by pushing approximately $5 \mathrm{~mL}$ of filtered ethanol through them. The particle samples accumulated in the filtered ethanol were dried in a vacuum drying oven $\left(30^{\circ} \mathrm{C}\right)$, after which the samples were diluted to $2 \mathrm{~mL}$ with filtered ethanol in a glass measuring flask and mixed for subsequent analysis.

\section{Sample analysis}

One microlitre of each sample collected with the procedure described above was analysed by the laser direct infrared 
(LDIR) chemical imaging system (Agilent 8700 ) to identify the number of particles and the polymer types and sizes of the collected particles. Notably, the LDIR chemical imaging system applied in this research relies on $\mu$-FTIR technology, and the detection limit is $>20 \mu \mathrm{m}$ (Pico et al. 2019). This means that nano- or microplastics with sizes smaller than $20 \mu \mathrm{m}$ are not detectable by the LDIR chemical imaging system. Considering that smaller particles were possibly collected during sampling, transmission electron microscopy (TEM, JEM100CXII, JEOL, Ltd., Japan) was utilized to detect any nanosized particles.

\section{Statistical analysis}

Experimental data were collected and analysed using SigmaPlot 12.5 and were presented as the mean \pm standard deviation from three replicates.

\section{Results and discussion}

\section{Microparticles release from daily plastic feeding and water bottles}

After 100 opening/closing cycles, large microparticles were released from the feeding bottle caps and necks, as shown in Table 1. The highest number of microparticles released was 393 ( \pm 57.5) particles/mL for FB-Brand 1, and the lowest number of microparticles released was $53( \pm 9.4)$ particles/ $\mathrm{mL}$ for FB-Brand 3. The lowest release number could be explained by the differences in glass and plastic bottle bodies and brands. FBs bottles with plastic bodies tended to release approximately 2 times more microparticles than FBs with glass bodies (Table 1). Additionally, the different brands showed various levels of microparticle release after 100 opening/closing cycles; for example, FB-Brand 1 (plastic bottle body) released more than 3 times more microparticles than FB-Brand 2 (plastic bottle). The influence of brand on microparticle release was detected previously and attributed to the different cap abrasions occurring for different brands of plastic water bottles (Winkler et al. 2019).

After 100 opening/closing cycles, the total numbers of released microparticles ranged from $100( \pm 23.3)$ to $209( \pm 38.4)$ particles $/ \mathrm{mL}$, as shown in Table 1 . Significantly more particles were released from WBs with plastic bodies than from WBs with glass bodies $(p<0.01)$. This trend also prevailed for FBs during comparison of FB-Brand 2 to FB-Brand 3. These results clearly indicated that, in addition to plastic caps, plastic bottle necks are another source of particle release during daily bottle usage. Interestingly, the brand influence on microparticle release was not significant for WBs, as shown by the comparable particle release numbers (Table 1).
Inspection of Fig. 1A shows a good fit $\left(r^{2}=0.811\right)$ and suggests that the total numbers of microparticles released from FBs and WBs were highly related to abrasion distance. The outlier was FB-Brand 1, as shown by its relatively high particle release number $(393 \pm 57.5$ particles $/ \mathrm{mL})$, which could be attributed to its thick neck and plastic body. Indeed, the long abrasion distance tended to enhance particle release and FBs ageing. However, the numbers of MPs identified were independent of the abrasion distance, as shown in Fig. 1B $\left(r^{2}=\right.$ $0.789, p=0.160$ with the FB-Brand 1 as an outlier; $r^{2}=$ $0.0003, p=0.769$ without the FB-Brand 1 outlier). This result was inconsistent with those in previous reports. It was likely that older or legacy of polypropylene FBs released larger amounts of particles than new FBs (Sobhani et al. 2020), and almost 8.5 times more plastic particles were released from reusable plastic bottles than single-use plastic bottles (Schymanski et al. 2018); moreover, increasing MPs release was observed after a series of bottle opening/closing cycles ranging from 1 to 100 (Winkler et al. 2019), as shown in Fig. 3. This different result could be explained by the different methods applied. For example, the LDIR chemical imaging system applied in this research relied on $\mu$-FTIR technology, and the detection limit was $>20 \mu \mathrm{m}$ (Pico et al. 2019), which means that nano- or microplastics smaller than $20 \mu \mathrm{m}$ were undetectable. Therefore, some nanosized plastic particles were excluded from the linear regression, as shown in Fig. 2 and Fig. 3.

\section{Type, size and morphology of MPs}

Based on the product information (Table S1) and the results of determinations from the $\mu$-FTIR and LDIR chemical imaging systems, the polymer types identified were polycarbonate (PC) and polypropylene (PP) for WBs and polyphenylene sulfone resins (PPSU) for FBs. In addition to PC, PP and PPUS, identified microplastics also included polyamide, polychloroethylene, polyurethane, polyethylene terephthalate, polystyrene and polyethylene (Fig. S2), which accounted for relatively low percentages (from 6.6 to 18.4\%) of the observed MPs. These additional polymer types were likely bound to the FBs and/or WBs during the production process and were released during the sampling processes (e.g. opening/closing abrasion). Various MPs sizes and shapes were observed for the feeding bottle and water bottle samples. According to data from the LDIR chemical imaging system, the detected MPs exhibited sizes ranging from 20 to $500 \mu \mathrm{m}$, with a large percentage of sizes ranging from 20 to $100 \mathrm{~nm}$, which was consistent with the trend for total detected microparticles (Fig. 2 and Fig. 3B, D, E). In the TEM images of particle shapes, cubic, spherical and irregular shapes were observed, which was consistent with the results of Fadare et al. in studies of plastic food containers (Fadare et al. 2020). Different sizes, shapes and types of nano- and microplastics impart various 
Table 1 Basic information on the particles collected for each WB- and FB-Brand and injectors. The data are from triplicate analyses

\begin{tabular}{|c|c|c|c|c|c|c|}
\hline & $\begin{array}{l}\text { Outer diameter } \\
(\mathrm{cm})\end{array}$ & Total microparticles (particles $/ \mathrm{mL}$ ) & $\begin{array}{l}\text { Microplastics } \\
\text { (particles/mL) }\end{array}$ & $\begin{array}{l}\text { Cellulosics }(\%) \\
\text { (particles/mL) }\end{array}$ & $\begin{array}{l}\text { Additives (\%) } \\
\text { (particles/mL) }\end{array}$ & $\begin{array}{l}\text { Others } \\
\text { (particles } / \mathrm{mL} \text { ) }\end{array}$ \\
\hline FB-Brand 1 & $5.45( \pm 0.06)$ & $393( \pm 57.5)$ & $112(28.6 \%)$ & $13(3.4 \%)$ & n.a. & $268(68.0 \%)$ \\
\hline FB-Brand 2 & $4.60( \pm 0.06)$ & $127( \pm 33.4)$ & $53(42.1 \%)$ & n.a. & $30(23.7 \%)$ & $44(34.2 \%)$ \\
\hline FB-Brand 3 & $5.30( \pm 0.08)$ & $53( \pm 9.4)$ & $10(18.8 \%)$ & $3(6.3 \%)$ & n.a. & $40(74.9 \%)$ \\
\hline WB-Brand 1 & $4.65( \pm 0.03)$ & $197( \pm 46.5)$ & $15(7.5 \%)$ & $19(9.8 \%)$ & $142(72.2 \%)$ & $21(10.5 \%)$ \\
\hline WB-Brand 2 & $3.50( \pm 0.01)$ & $209( \pm 38.4)$ & $37(17.8 \%)$ & $29(14.0 \%)$ & $121(58.0 \%)$ & $22(10.2 \%)$ \\
\hline WB-Brand 3 & $6.50( \pm 0.04)$ & $127( \pm 27.6)$ & $33(26.2 \%)$ & $13(10.5 \%)$ & $23(18.4 \%)$ & $58(44.9 \%)$ \\
\hline WB-Brand 4 & $6.60( \pm 0.04)$ & $100( \pm 23.3)$ & $13(13.3 \%)$ & n.a. & $30(30.0 \%)$ & $57(56.7 \%)$ \\
\hline Injector & $1.40( \pm 0.01)^{a}$ & $174( \pm 20.3)^{b}$ & $34(19.3 \%)$ & $6(3.8 \%)$ & n.a. & $134(76.9 \%)$ \\
\hline
\end{tabular}

n.a. means the items were not observed with the LDIR chemical imaging system

${ }^{a}$ denotes the inner diameters of injectors

${ }^{b}$ denotes that microparticles were collected from 100 injectors and with three replicates, and thus the unit is particles/100 injectors

hazardous effects on infants and children. Since the bodies of babies and children do not have sufficient defence mechanisms, it is easy for microplastics to enter their bodies through the oral cavity, skin or other pathways, causing different degrees of impact. Therefore, the risk of microplastics to children should not be underestimated.

\section{Other MPs}

Based on the technical limitations of the LDIR chemical imaging system, nano- or microplastics with sizes smaller than $20 \mu \mathrm{m}$ were undetectable. Therefore, the collected samples were also examined through TEM, as shown in Fig. 3. Many micro- or nanosized particles were detected for these samples, regardless of whether they were plastic or glass bottles or offered by different manufacturers. For example, numerous nanosized particles were found from FB-Brand 2 and WB-Brand 1 bottles, and they displayed cubic, spherical and irregular shapes. Moreover, the possible hazardous effects for infants or children induced by nanosized particles with

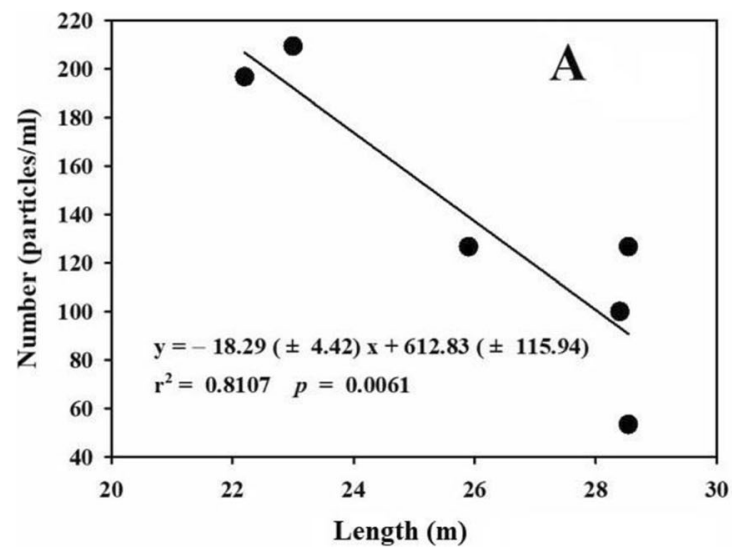

Fig. 1 A Linear regressions for total detected particles (particles $/ \mathrm{mL}$ ) vs. abrasion distance $(\mathrm{m})$, and the outlier (FB-Brand 1 ) is not shown in the figure; B Linear regressions for detected MPs (particles $/ \mathrm{mL}$ ) vs. abrasion multiple particle shapes are derived from recent toxicological data on aquatic biota. Interestingly, cellulosic particles were detected in up to $71 \%$ of these FBs and WBs samples. Plastic additives are other sources of particle types in plastics. For example, calcium stearate and silicone are two commonly used additives for plastic products and were found in FBs and WBs. According to proven toxicological data (Cutting 1952), hazardous effects possibly result from nanosized calcium stearates and silicones during the daily use of FBs and WBs. The "others" items listed in Table 1 suggested that particles from some nonplastic materials were detected, and a higher proportion was detected in glass bottles than in plastic bottles. In the process of opening and closing FBs and WBs, and due to increasing wear and tear and plastic aging, some plastic additives and glass particles are released, and the main components of these particles are likely to be silicates and stearates. Therefore, the "others" items may be primarily composed of silica and some chitin and/or coal, suggesting that glass fragments could be another source of potential hazards.

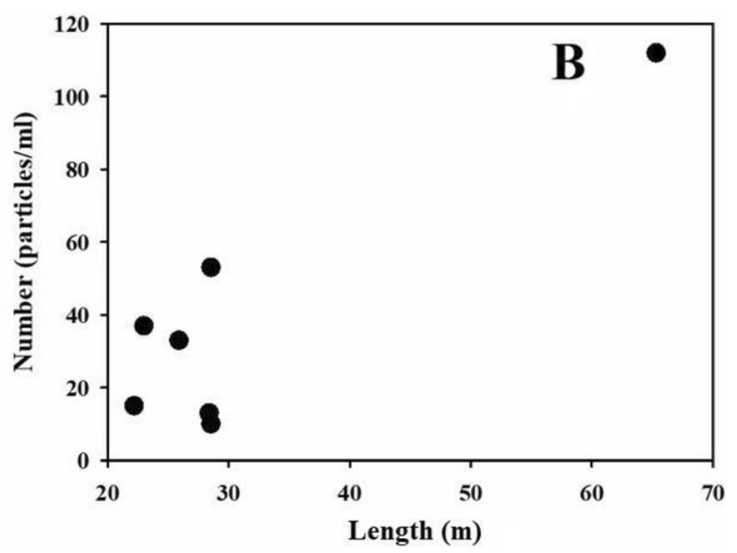

distance $(\mathrm{m})\left(y=2.11( \pm 0.49) x-27.82( \pm 16.87), r^{2}=0.789, p=0.160\right)$, and the point in the top right corner represents the outlier (FB-Brand 1) 
Fig. 2 Particle size distribution for all particles collected from FBs, WBs and injectors. A FBBrand 1; B FB-Brand 2; C FBBrand 3; D Injector; E WB-Brand 1; F WB-Brand 2; G WB-Brand 3; H WB-Brand 4
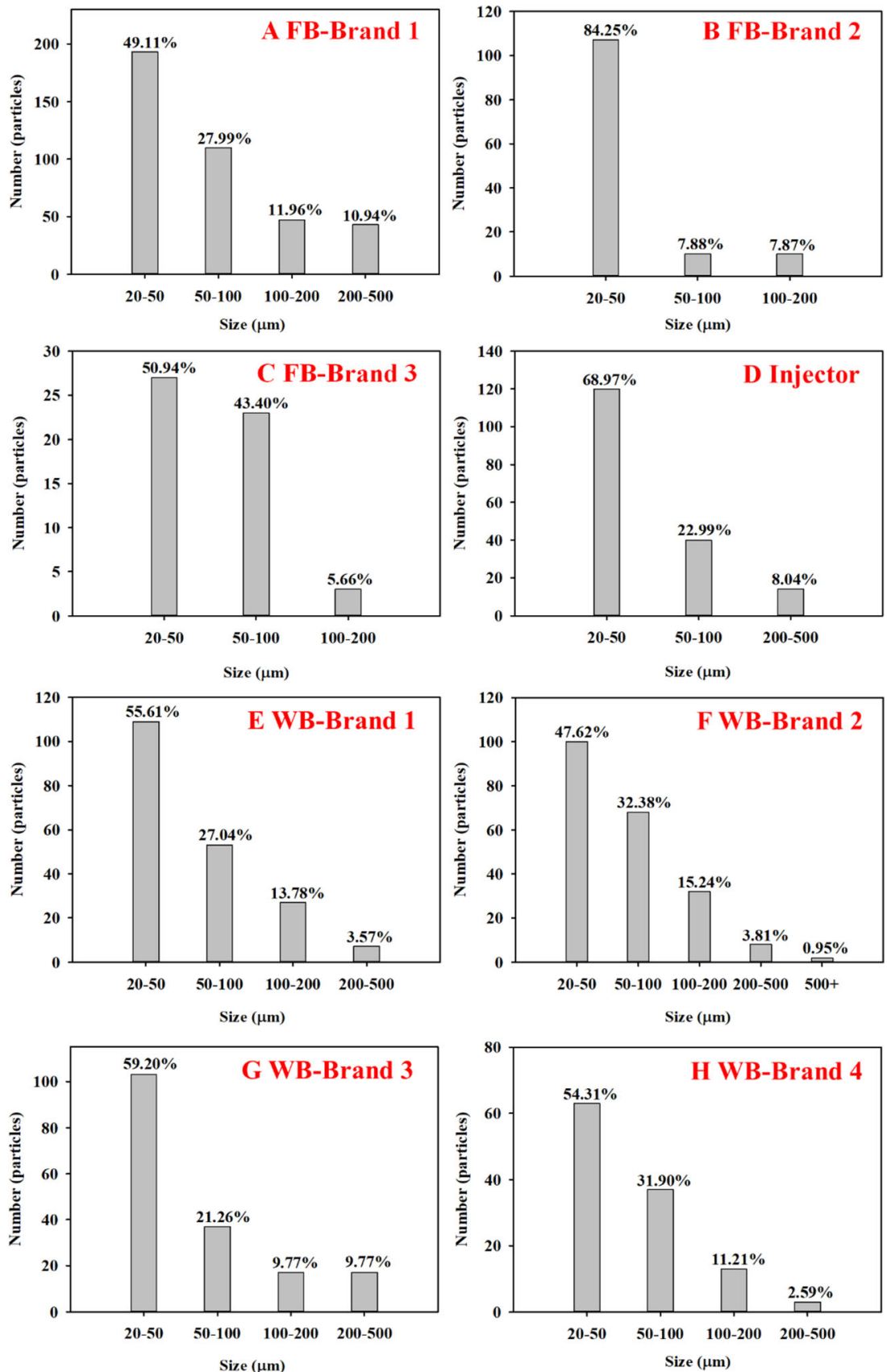

\section{Risk of MPs release from injectors}

Plastic injectors are also used extensively in daily life. For example, insulin and antibiotics are injected intramuscularly and intravenously into diabetic patients and livestock farms by utilizing large numbers of single-use plastic injectors. Intravenous injection increases the chance of direct contact between microplastics and the body. Statistically, 1.74 MPs particles were released from every use of a single-use plastic injector. In addition, the released microplastics have larger specific surface areas and a smaller particle sizes than other sources, which makes it easy to absorb the injected drugs and enter human blood vessels. Additive particles seen in FBs and WBs, such as calcium stearate and silicone, were not observed with injectors. Indeed, polypropylene bodies and polystyrene plugs are rarely introduced as additive particles. The polymer type identified for single-use plastic injectors was polypropylene, which matches the product information. Nevertheless, there are some nano- and/or microsized $(<20 \mu \mathrm{m})$ particles released from injectors, as shown in Fig. 3I. These small particles showed cubic, spherical and irregular shapes, which is consistent with those found from FBs and WBs. The results suggested that these plastic injectors could be a direct source of MPs introduction and thereby impart risks to human health. 


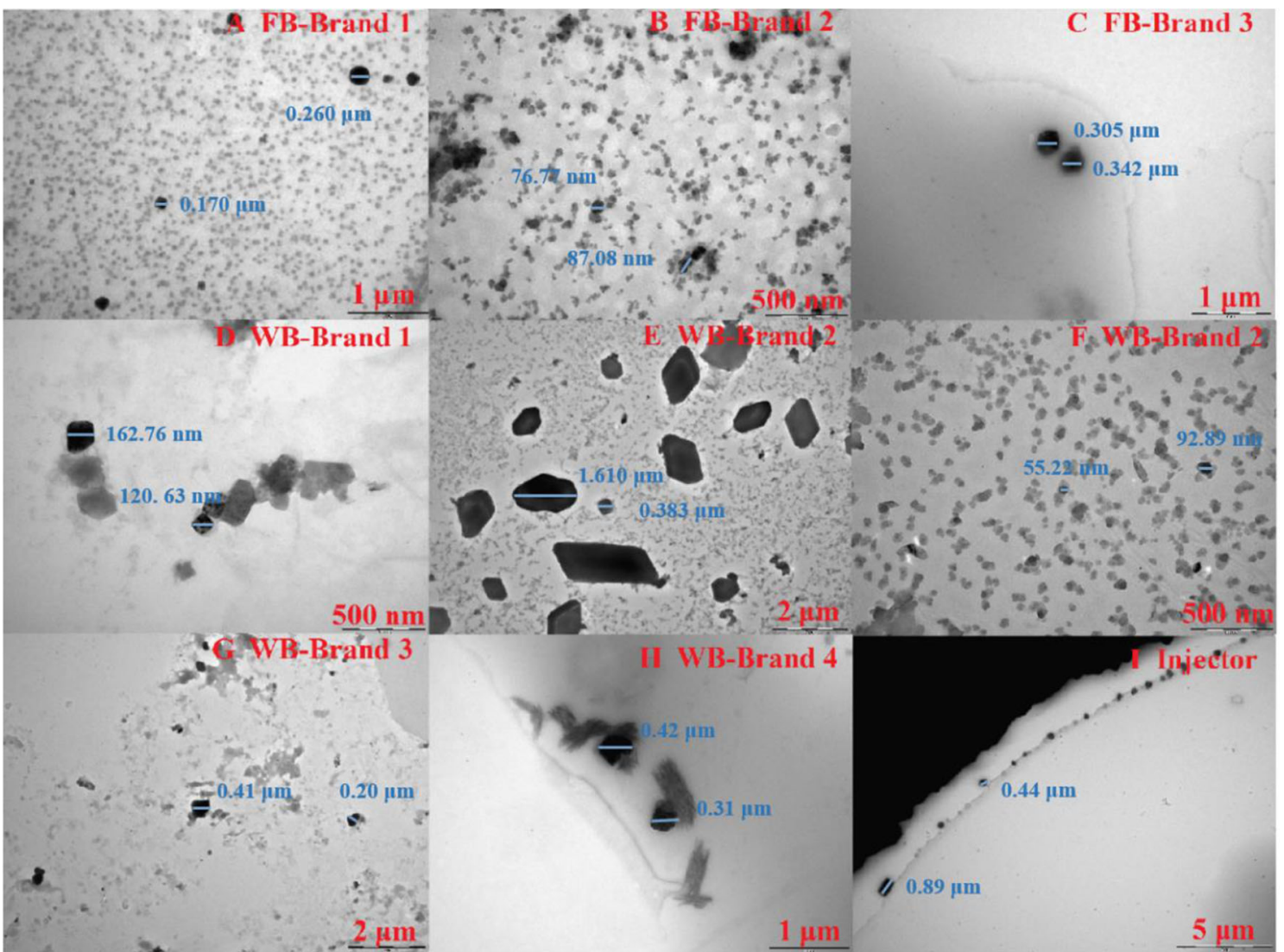

Fig. 3 TEM images of particles from FBs, WBs and injectors. A FB-Brand 1; B FB-Brand 2; C FB-Brand 3; D WB-Brand 1; E WB-Brand 2; F WBBrand 2; G WB-Brand 3; H WB-Brand 4; I Injector

\section{Risk of infant and child exposure to MPs from FBs and WBs}

To date, these selected FBs and WBs exhibit good sales, as shown for FB-Brand 1 (2981 per month) and WB-Brand 3 (1049 per month), which indicates that large numbers of these FBs and WBs were used by Chinese infants and children. Indeed, the 6.13 billion Renminbi (RMB) retail sales of milk bottles (2019) suggested that FBs and WBs are widely used by most Chinese families (Network 2020). In particular, there is a greater demand for bottles and glasses in families with two children, which also promotes the use of feeding bottles and water bottles. Under certain circumstances, the test with 100 opening/closing cycles simulated approximately three months of usage and indicated that approximately 117.3 MPs and 16.3 MPs $(>20 \mu \mathrm{m})$ per month would be ingested by infants and children from use of FBs and WBs, respectively. Moreover, MPs and other particles, such as calcium stearate and silicone, with sizes $<20 \mu \mathrm{m}$ could also be extensively ingested by infants and children. On the one hand, microplastics and other microparticles are released from FBs and WBs through opening and closing cycles. Microplastics easily combine with the soluble components in milk powder when infants and children consume milk powder and water, so infants and children inevitably ingest microplastics and microparticles. On the other hand, the microparticles produced by abrasion eventually enter environmental water systems, inevitably causing secondary pollution and increasing the risks for infants and children when they drink these powdered products. Therefore, based on the certain numbers of MPs and the uncertain numbers of nanoplastics released from these FBs and WBs, Chinese infants and children are suffering possible threats from these released plastic particles and other particles. Moreover, even when adequate washing is performed, these micro- and nanosized plastics arrive at wastewater treatment plants. Although MPs may be effectively removed by wastewater treatment plants, a significant number of MPs enter the receiving water system with the effluent or/and are released into the soil environment by application of sludge to land parcels (Sun et al. 2019), wherein their fate and transport modes are still unknown.

\section{Conclusion}

This study indicated that FBs, WBs and injectors are potential sources for release of micro- and nanosized plastics during daily usage. Various amounts, sizes and shapes of plastics 
were observed by TEM and LDIR chemical imaging systems, suggesting that the plastics released from FBs and WBs could present uncertain hazardous effects on the health of Chinese infants and children. Based on the good linear regressions for plots of total particles vs. abrasion distance $\left(r^{2}=0.811\right)$, thinnecked glass bottles release fewer microparticles than thicknecked plastic bottles, and bottles could be safer for daily use by infants or children. After considering the impacts of brands and plastic bodies on levels of microparticle release, highquality bottles (high prices) and glass bodies for FBs and WBs are suggested for daily use by infants and children. Our data provide additional evidence indicating that babies and children could be exposed to MPs through daily FBs and WBs usage. Additionally, injection of medicines with plastic injectors directly increases the exposure of patients to MPs. Therefore, the health risks of micro- and nanosized plastics for humans, especially babies and children, and for the environment should be seriously considered.

Author contribution Ke Song: Writing - original draft, formal analysis. Runrun Ding and Caiyun Sun: Writing - review and editing. Lunguang Yao: Writing - Review and formal analysis. Weicheng Zhang: Conceptualization and supervision.

Funding The study was supported by the National Natural Science Foundation of China-Henan Joint Fund (No. U1804109), the Foundation of Henan Educational Committee (No. 19A330003; No. 18A230011), Science and Technology Program of Henan Department of Science and Technology (No. 192102310305; No. 182102310077) and the Foundation of Nanyang Normal University (No. 2018ZX023).

Data availability All data generated or analysed during this study are included in this published article.

\section{Declarations}

Ethics approval and consent to participate Not applicable. (The article does not address ethical issues.)

Consent for publication Not applicable. (Personal privacy and data are not included in the manuscript.)

Conflict of interest The authors declare no competing interests.

\section{References}

Alimba CG, Faggio C (2019) Microplastics in the marine environment: current trends in environmental pollution and mechanisms of toxicological profile. Environ Toxicol Pharmacol 68:61-74. https://doi. org/10.1016/j.etap.2019.03.001

Aragaw TA (2020) Surgical face masks as a potential source for microplastic pollution in the COVID-19 scenario. Mar Pollut Bull 159:111517. https://doi.org/10.1016/j.marpolbul.2020.111517

Botterell ZLR, Beaumont N, Dorrington T, Steinke M, Thompson RC, Lindeque PK (2019) Bioavailability and effects of microplastics on marine zooplankton: a review. Environ Pollut 245:98-110. https:// doi.org/10.1016/j.envpol.2018.10.065
Burgos-Aceves MA, Abo-Al-Ela HG, Faggio C (2021) Physiological and metabolic approach of plastic additive effects: immune cells responses. J Hazard Mater 404:124114. https://doi.org/10.1016/j. jhazmat.2020.124114

Busse K, Ebner I, Humpf H-U, Ivleva N, Kaeppler A, Oßmann BE, Schymanski D (2020) Comment on "Plastic Teabags Release Billions of Microparticles and Nanoparticles into Tea". Environ Sci Technol 54:14134-14135. https://doi.org/10.1021/acs.est. $0 \mathrm{c} 03182$

Cutting WC (1952) Toxicity of silicones. Stanford Med Bull 10:23-26

Danopoulos E, Jenner LC, Twiddy M, Rotchell JM (2020) Microplastic contamination of seafood intended for human consumption: a systematic review and meta-analysis. Environ Health Perspect 128: 126002. https://doi.org/10.1289/EHP7171

Ding R, Tong L, Zhang W (2021) Microplastics in freshwater environments: sources, fates and toxicity. Water Air Soil Pollut 232:181. https://doi.org/10.1007/s11270-021-05081-8

Du J, Xu S, Zhou Q, Li H, Fu L, Tang J, Wang Y, Peng X, Xu Y, Du X (2020) A review of microplastics in the aquatic environmental: distribution, transport, ecotoxicology, and toxicological mechanisms. Environ Sci Pollut Res Int 27:11494-11505. https://doi.org/10. 1007/s11356-020-08104-9

Fadare OO, Wan B, Zhao L, Guo LH (2020) Microplastics from consumer plastic food containers: are we consuming it? Chemosphere. 253: 126787. https://doi.org/10.1016/j.chemosphere.2020.126787

Gatidou G, Arvaniti OS, Stasinakis AS (2019) Review on the occurrence and fate of microplastics in Sewage Treatment Plants. J Hazard Mater 367:504-512. https://doi.org/10.1016/j.jhazmat.2018.12.081

Guzzetti E, Sureda A, Tejada S, Faggio C (2018) Microplastic in marine organism: environmental and toxicological effects. Environ Toxicol Pharmacol 64:164-171. https://doi.org/10.1016/j.etap.2018.20009

Hernandez LM, Xu EG, Larsson HCE, Tahara R, Maisuria VB, Tufenkji N (2019) Plastic teabags release billions of microparticles and nanoparticles into tea. Environ Sci Technol 53:12300-12310. https://doi. org/10.1021/acs.est.9b02540

Hernandez LM, Xu EG, Larsson HCE, Tahara R, Maisuria VB, Tufenkji N (2020) Response to Comment on "Plastic Teabags Release Billions of Microparticles and Nanoparticles into Tea". Environ Sci Technol 54:14136-14137. https://doi.org/10.1021/acs.est. 0c06422

Horton AA, Jürgens MD, Lahive E, van Bodegom PM, Vijver MG (2018) The influence of exposure and physiology on microplastic ingestion by the freshwater fish Rutilus rutilus (roach) in the River Thames. UK Environ Pollut 236:188-194. https://doi.org/10.1016/j. envpol.2018.01.044

Li D, Shi Y, Yang L, Xiao L, Kehoe DK, Gun'ko YK, Boland JJ, Wang JJ (2020) Microplastic release from the degradation of polypropylene feeding bottles during infant formula preparation. Nat Food 1: 746-754. https://doi.org/10.1038/s43016-020-00171-y

Nelms SE, Galloway TS, Godley BJ, Jarvis DS, Lindeque PK (2018) Investigating microplastic trophic transfer in marine top predators. Environ Pollut 238:999-1007. https://doi.org/10.1016/j.envpol. 2018.07.051

Network CIR 2020: Research Report on milk bottle Market in 2020

Pagano M, Vazzana I, Gentile A, Caracappa G, Faggio C (2019) Hematological and biochemical parameters in Sea turtles (Caretta caretta) after stranding. Reg Stud Mar Sci 32:100832. https://doi. org/10.1016/j.rsma.2019.100832

Peixoto D, Pinheiro C, Amorim J, Oliva Teles L, Guilhermino L, Vieira N (2019) Microplastic pollution in commercial salt for human consumption: a review. Estuar Coast Shelf Sci 219:161-168. https://doi. org/10.1016/j.ecss.2019.02.018

Pico Y, Alfarhan A, Barcelo D (2019) Nano- and microplastic analysis: focus on their occurrence in freshwater ecosystems and remediation technologies. Trends Anal Chem 113:409-425. https://doi.org/10. 1016/j.trac.2018.08.022 
Prata JC, da Costa JP, Duarte AC, Rocha-Santos T (2019) Methods for sampling and detection of microplastics in water and sediment: a critical review. Trends Anal Chem 110:150-159. https://doi.org/10. 1016/j.trac.2018.10.029

Prokić MD, Radovanović TB, Gavrić JP, Faggio C (2019) Ecotoxicological effects of microplastics: examination of biomarkers, current state and future perspectives. Trends Anal Chem 111:37-46. https://doi.org/10.1016/j.trac.2018.12.001

Prokić MD, Gavrilović BR, Radovanović TB, Gavrić JP, Petrović TG, Despotović SG, Faggio C (2021) Studying microplastics: lessons from evaluated literature on animal model organisms and experimental approaches. J Hazard Mater 414:125476. https://doi.org/10. 1016/j.jhazmat.2021.125476

Rillig MC, Bonkowski M (2018) Microplastic and soil protists: a call for research. Environ Pollut 241:1128-1131. https://doi.org/10.1016/j. envpol.2018.04.147

Schwabl P, Kppel S, Knigshofer P, Bucsics T, Liebmann B (2019) Detection of various microplastics in human stool: a prospective case series. Ann Intern Med 171:453-457

Schymanski D, Goldbeck C, Humpf H-U, Fürst P (2018) Analysis of microplastics in water by micro-Raman spectroscopy: release of plastic particles from different packaging into mineral water. Water Res 129:154-162. https://doi.org/10.1016/j.watres.2017.11. 011

Senathirajah K, Attwood S, Bhagwat G, Carbery M, Wilson S, Palanisami T (2021) Estimation of the mass of microplastics ingested - a pivotal first step towards human health risk assessment. J Hazard Mater 404:124004. https://doi.org/10.1016/j. jhazmat.2020.124004

Shruti VC, Pérez-Guevara F, Elizalde-Martínez I, Kutralam-Muniasamy G (2021) Toward a unified framework for investigating micro(nano)plastics in packaged beverages intended for human consumption. Environ Pollut 268:115811. https://doi.org/10.1016/j. envpol.2020.115811

Sobhani Z, Lei Y, Tang Y, Wu L, Zhang X, Naidu R, Megharaj M, Fang C (2020) Microplastics generated when opening plastic packaging. Sci Rep 10:4841. https://doi.org/10.1038/s41598-020-61146-4
Strungaru S-A, Jijie R, Nicoara M, Plavan G, Faggio C (2019) Micro(nano) plastics in freshwater ecosystems: abundance, toxicological impact and quantification methodology. Trends Anal Chem 110: 116-128. https://doi.org/10.1016/j.trac.2018.10.025

Sun C, Zhang W, Ding R, Wang J, Yao L (2020) Mechanism of low concentrations of polystyrene microplastics influence the cytotoxicity of $\mathrm{Ag}$ ions to Escherichia coli. Chemosphere. 253:126705. https://doi.org/10.1016/j.chemosphere.2020.126705

Sun J, Dai X, Wang Q, van Loosdrecht MCM, Ni B-J (2019) Microplastics in wastewater treatment plants: detection, occurrence and removal. Water Res 152:21-37. https://doi.org/10.1016/j. watres.2018.12.050

Uhl W, Eftekhardadkhah M, Svendsen C (2018): Mapping microplastic in Norwegian drinking water, Norsk. Vann. Report. 241/2018

Watts AJ, Urbina MA, Corr S, Lewis C, Galloway TS (2015) Ingestion of plastic microfibers by the crab Carcinus maenas and its effect on food consumption and energy balance. Environ Sci Technol 49: 14597-14604. https://doi.org/10.1021/acs.est.5b04026

Winkler A, Santo N, Ortenzi MA, Bolzoni E, Bacchetta R, Tremolada P (2019) Does mechanical stress cause microplastic release from plastic water bottles? Water Res 166:115082. https://doi.org/10.1016/j. watres.2019.115082

Xiong X, Zhang K, Chen X, Shi H, Luo Z, Wu C (2018) Sources and distribution of microplastics in China's largest inland lake Qinghai Lake. Environ Pollut 235:899-906. https://doi.org/10. 1016/j.envpol.2017.12.081

Zhang Q, Xu EG, Li J, Chen Q, Ma L, Zeng EY, Shi H (2020) A review of microplastics in table salt, drinking water, and air: direct human exposure. Environ Sci Technol 54:3740-3751. https://doi.org/10. 1021/acs.est.9b04535

Publisher's note Springer Nature remains neutral with regard to jurisdictional claims in published maps and institutional affiliations. 